\title{
Breit wirksam im Kampf gegen Entzündungen
}

Studien zeigen häufig einen Vitamin-C-Mangel bei Krebs, chronisch-entzündlichen Erkrankungen und aktuellen Herausforderungen wie COVID-19 und Long-COVID. Die antioxidative, antientzündliche, endothelschützende und immunmodulierende Wirkung von Vitamin C erklärt dessen therapeutische Möglichkeiten bei diesen Erkrankungen.

Umwelttoxische Belastungen durch Metalle, Pestizide/Insektizide, Flammschutz- und Lösungsmittel können zu chronischen Erkrankungen mit zunehmend gestörter Immuntoleranz führen, konstatiert Dr. Astrid Kohl, Fachärztin für Innere Medizin und Naturheilverfahren in Berlin. Fallberichte zu Long-COVID, rezidivierenden HerpesInfektionen, Multiorganbeschwerden mit Osteoporose, Schilddrüsen-, Magen- und Darmerkrankungen aber auch zu Krebserkrankungen zeigen die ursächliche Beteiligung von neurotoxischen Insektiziden oder toxischen Belastungen in der Zahnmedizin und bei Gelenk-Implantaten. Als wichtigste Maßnahme, um die Entzündungskaskade zu durchbrechen und die individuelle Immuntoleranz und Regulationsfähigkeit wiederherzustellen, nennt Kohl hochdosiertes, intravenöses Vitamin C in Verbindung mit einer angepassten orthomolekularen Therapie und einer Expositionsminderung bzw. -vermeidung.

Seine Bedeutung für das adaptive und angeborene Immunsystem und vor allem die anti-oxidative und damit anti-entzündliche Wirkung erkläre auch, so Dr. Andreas Pinter, Klinik für Dermatologie, Venerologie und Allergologie am Universitätsklinikum Frankfurt, das breite Anwendungspotenzial von Vitamin C. Dieses umfasse entzündliche Hauterkrankungen ebenso, wie chronisch-entzündliche Darmerkrankungen, rheumatoide Arthritis, Psoriasis-Arthritis und Depressionen. Entzündung und oxidativer Stress stehen gleichfalls im Fokus der Pathomechanismen einer Sars-CoV-2 Infektion. Als wichtiger Immunmodulator ist Vitamin C essenziell für die antivirale T-Zellantwort und schützt gleichzeitig vor dem Zytokin-Sturm bei COVID-19. Wichtig sei, intravenöses Vitamin C gleich zu Beginn eines Infektes einzusetzen, betont Dr. Ruth Poglitsch, Allgemeinmedizinerin und Assistenzärztin für Anästhesiologie und Intensivmedizin in Graz. Falldokumentationen zeigen auch die positive Wirkung von intravenösem Vitamin C bei Patienten mit Long-COVID-Syndrom, die unter Fatigue, Atemnot und kognitiven Defiziten leiden.

\section{Potenzial bei Krebs}

Hochdosiertes Vitamin C bietet laut Studienevidenz auch chemotherapeutisches Potenzial bei Krebs. Experimentelle Studien zeigen beispielsweise beim Glioblastom eine selektive, pro-oxidative und damit antikanzerogene Wirkung von supportivem hochdosiertem, intravenösem Vitamin C und eine Sensitivierung für die Radiochemotherapie. Die Verträglichkeit ist ersten Patientendaten zufolge gut und die Lebenszeit kann verlängert werden, berichtete Prof. Dr. Sascha Venturelli von der Universität Hohenheim. Vitamin C weist MultitargetingEffekte auf, betonte Dr. Qi Chen. Die Assistenzprofessorin an der Universität Kansas war 2005 an der Entdeckung beteiligt, dass hohe Vitamin C-Konzentrationen über die Bildung von Wasserstoffperoxid selektiv tumorzytotoxisch wirken, aber gesunde Zellen nicht schädigen. Vitamin $C$ verbessert in der Tumormikroumgebung die Kollagenzusammensetzung und reduziert das Metastasierungsrisiko. Zudem verändert Vitamin C das epigenetische Profil in Richtung Tumorsuppression (HIF, TET2).

\section{Günstiger Einfluss bei Sepsis}

Die in 21 Ländern durchgeführte REMAP-CAP-Studie soll die optimale Behandlung für ambulant erworbene Lungenentzündungen bestimmen und kontinuierlich aktualisieren. COVID-19-Patienten werden, so Prof. Dr. Frank Brunkhorst vom Universitätsklinikum Jena, seit vergangenem Jahr explizit eingeschlossen und der Einsatz von intravenösem Vitamin C $(50 \mathrm{mg} /$ $\mathrm{kg} \mathrm{KG}$ alle 6 Stunden) wird seit heuer evaluiert. Die klinische Überlegung: Entzündungen und oxidativer Stress zählen zu den Mechanismen, die am häufigsten Organschäden bei Sepsis und COVID-19 verursachen. Vitamin C begrenzt die Produktion von Sauerstoffradikalen, stellt die Endothelfunktion wieder her und ist wichtiger Kofaktor bei der Synthese von Noradrenalin, Cortisol und Vasopressin. Deshalb sind niedrige Vitamin C-Spiegel mit Organversagen und dem Tod von COVID-19-Patienten assoziiert. Die Evidenz, dass Vitamin C den Verlauf bei Sepsis günstig beeinflusst, vor allem wenn es für drei bis vier Tage appliziert wird, ist groß.

Hinweis des Verlags. Der Verlag bleibt in Hinblick auf geografische Zuordnungen und Gebietsbezeichnungen in veröffentlichten Karten und Institutsadressen neutral.

Wien klin Mag 2021 ·24:139 https://doi.org/10.1007/s00740021-00407-y

๑) Springer-Verlag GmbH Austria, ein Teil von Springer Nature 2021
Quelle: Presseaussendung 3. Internationaler Kongress ${ }_{\text {,Hoch- }}$ dosiertes Vitamin C" von Pascoe Naturmedizin 